\title{
Relação entre alterações climáticas e fatores determinantes da mortalidade de idosos no município de São Carlos (SP) em um período de dez anos
}

\author{
Relationship between climate change and determinant factors \\ of mortality among the elderly in the municipality of São Carlos \\ (São Paulo, Brazil) over a period of ten years
}

Fabiana Vieira Soares ${ }^{1}$

Patrícia Greve ${ }^{1}$

Francisco Alburquerque Sendín ${ }^{1}$

Benedito Galvão Benze ${ }^{1}$

Alessandra Paiva de Castro ${ }^{1}$

José Rubens Rebelatto ${ }^{1}$

${ }^{1}$ Departamento de Fisioterapia, Universidade Federal de São Carlos. Rodovia Washington Luís km 235. 13565-905 São Carlos SP.

bianasoares@hotmail.com

\begin{abstract}
The aim of this study was to identify the correlation between the number of deaths of elderly people and climate change in the district of São Carlos (SP) over a period of 10 years (19972006). Records of deaths were obtained from DATASUS for people aged over 60 who died between 1997 and 2006 in São Carlos. The average monthly maximum and minimum temperature data and relative air humidity in São Carlos were provided by the National Institute of Meteorology. The mortality coefficient of the district was calculated by gender and age and the resulting data were analyzed using t test, one-way ANOVA, the Bonferroni test and the Pearson correlation coefficient test. There were 8,304 deaths which predominantly occurred among males aged over 80 , and diseases of the circulatory system were the main cause of death. There was a positive correlation between mortality by infectious disease and minimum humidity, and a negative correlation between mortality by infectious diseases and minimum temperatures, between mortality caused by respiratory disease and minimum humidity, between mortality caused by endocrine disease and minimum and maximum temperature. Thereby, it was possible to conclude that there was a correlation between climate change and mortality among elderly individuals in São Carlos.
\end{abstract}

Key words Climate change, Elderly, Mortality
Resumo O objetivo desse estudo foi identificar as correlações existentes entre o número de óbitos de indivíduos idosos e as alterações climáticas no município de São Carlos (SP) em um período de 10 anos (1997-2006). Foram colhidas informações do registro de óbitos por meio do DATASUS, de pessoas com 60 anos ou mais, falecidas entre 1997 e 2006 no município. Os dados mensais da média das Temperaturas Máxima, Média e Minima e da Umidade Relativa do Ar no município de São Carlos foram fornecidos pelo Instituto Nacional de Meteorologia. Calcularam-se os coeficientes de mortalidade do municipio, por sexo e faixa etária e os dados foram analisados com teste $t$, ANOVA de fator único, teste de Bonferroni e teste de Pearson. Ocorreram 8.304 óbitos, com predominância de indivíduos na faixa dos 80 anos ou mais, do sexo masculino. As doenças do aparelho circulatório foram as principais causas de óbito. Houve correlação positiva entre mortalidade por doenças infecciosas e Umidade Minima e correlação negativa entre mortalidade por doenças infecciosas e Temperatura Minima Minima, entre mortalidade por doenças respiratórias e Umidade Mínima, entre mortalidade por doenças endócrinas e Temperatura Minima Minima e Temperatura Máxima Máxima. Dessa forma, foi possível concluir que houve relação entre o clima e a mortalidade de idosos em São Carlos.

Palavras-chave Alterações climáticas, Idoso, Mortalidade 


\section{Introdução}

Muitos meteorologistas e climatólogos afirmam que a ação humana está influenciando a ocorrência do aquecimento global. O Painel Intergovernamental para as Mudanças Climáticas (IPCC, em inglês), estabelecido pela Organização das Nações Unidas e pela Organização Meteorológica Mundial, em 1988, relata que o aquecimento observado durante os últimos 50 anos provavelmente se deve ao aumento do efeito estufa ${ }^{1}$. Há também evidências de que o aquecimento seja devido a atividades humanas que incluem maior uso de águas subterrâneas e uso do solo para a agricultura industrial, maior consumo energético e maior poluição $\mathrm{O}^{2}$. A principal evidência do aquecimento global vem das medidas de temperatura de estações meteorológicas em todo o globo desde 1860. No século XXI, caso as emissões de poluentes continuem nos níveis atuais, a temperatura da Terra deverá subir cerca de três graus Celsius e para os próximos vinte anos, é projetado um aumento de 0,2 graus Celsius por década ${ }^{3}$.

As condições atmosféricas exercem forte influência sobre a sociedade e sobre as condições de saúde, sendo as doenças do ser humano uma das manifestações dessa interação ${ }^{4}$. O risco de acidente vascular encefálico é acentuado em temperaturas acima de $25^{\circ} \mathrm{C}$ e doenças respiratórias como ataques de asma e febre do feno são frequentes no verão ${ }^{5}$. Tumores, como o câncer de pele, podem surgir e se desenvolver porque a radiação ultravioleta induz à formação de células T-supressoras que, por sua vez, inibem o mecanismo de defesa contra tumores ${ }^{6}$.

Segundo Beltrando e Chemery ${ }^{7}$, a manutenção do equilíbrio térmico do corpo com seu ambiente (homeotermia) é uma das principais exigências para o conforto e para a saúde e é regulada quase inteiramente por mecanismos nervosos de retroalimentação, os quais operam através dos centros reguladores da temperatura, localizados no hipotálamo ${ }^{8}$. A vulnerabilidade ao calor ocorre em pessoas com idade avançada devido às mudanças intrínsecas no sistema de termorregulação, como a diminuição da água corporal, a redução da taxa de suor e a menor eficiência do sistema cardiovascular-11, ou por causa da presença de drogas que interferem no sistema de homeostasia normal ${ }^{12,13}$.

Mortes, principalmente por doenças isquêmicas do coração, elevaram-se em sujeitos com idade igual ou superior a 65 anos durante as ondas de calor em 1972, 1973 e 1975 ocorridas na cidade de Nova Iorque ${ }^{14}$. Outros acometimen- tos, inclusive altas taxas de mortalidade, envolvendo preferencialmente os idosos, foram relatados durante curtos períodos de tempo quente durante o verão da cidade de Londres no período de 1965 a $1972^{15}$, em Birmingham no Reino Unido $^{16}$, em Portugal ${ }^{17}$, na Itália ${ }^{18}$, na República Checa $^{19}$ e na Califórnia ${ }^{20}$

Estudando os óbitos ocorridos na onda de calor da França no ano de 2003, Stöllberger et al. ${ }^{21}$ constataram que um quinto dos óbitos foi devido à combinação de desidratação, golpe de calor e hipertermia, e um décimo foi atribuído à desidratação. Levantou-se a hipótese de que a adaptação fisiológica ao calor não foi eficaz nas vítimas que estavam sob efeitos colaterais de certas drogas que prejudicariam a termorregulação e reprimiam a sede. Isto poderia explicar por que muitos morreram vítimas de desidratação, apesar da disponibilidade de água. Brooke e Bell ${ }^{22}$ estudaram 107 comunidades urbanas nos Estados Unidos da América e constataram que no período de 1987 a 2000 houve maior vulnerabilidade em idosos para mortes relacionadas ao clima, no entanto, outras faixas etárias mais jovens também foram atingidas.

Dessa forma, considerando as mudanças climáticas, seu possível impacto sobre a saúde dos idosos e a existência de uma estação meteorológica na cidade de São Carlos (São Paulo, Brasil), o objetivo do presente estudo foi identificar as correlações existentes entre o número de óbitos de indivíduos idosos, os fatores que os determinaram e as alterações climáticas no município de São Carlos em um período de 10 anos (1997-2006).

\section{Método}

\section{Fontes de informação}

Foram utilizadas informações constantes no registro de óbito de pessoas acima de 60 anos, de ambos os sexos e que faleceram entre 1997 e 2006 no município de São Carlos (São Paulo, Brasil). Também foram utilizadas informações sobre os aspectos climáticos nesse período na cidade de São Carlos. Foram fontes de informação: a) O Instituto Nacional de Meteorologia (INMET), que forneceu dados meteorológicos (Temperaturas Máxima, Média e Mínima e Umidade Relativa do Ar mensais), b) O Departamento de Informática do Sistema Único de Saúde (DATASUS) que disponibiliza informações dos registros de óbitos (número de óbitos e causa da morte de acordo com o Código Internacional de 
Doenças (CID-10), idade, sexo, estado civil, raça e ano de falecimento, e dados demográficos (população residente no município de São Carlos nos anos estudados).

\section{Procedimento}

Os valores mensais da Temperatura Média, da Temperatura Máxima, da Temperatura Mínima e da Umidade Relativa do Ar, referentes ao período de 1997 a 2006, foram utilizados para o cálculo das médias anuais. Também foram utilizadas as variáveis “Temperatura Máxima Máxima”, que se refere ao maior valor de temperatura máxima media mensal de cada ano; "Temperatura Mínima Mínima” (menor valor da temperatura mínima media mensal de cada ano); "Umidade Máxima" (maior valor da umidade media mensal de cada ano); e "Umidade Mínima" (menor valor da umidade media mensal de cada ano).

Foram obtidas informações sobre idade, sexo, ano de falecimento, número de óbitos, estado civil, raça e causa da morte de acordo com o CID10 dos indivíduos que faleceram, no período de 1997 a 2006, na cidade de São Carlos. Após a obtenção dessas informações na página na web do DATASUS (www.datasus.gov.br), elas foram correlacionadas entre si e com as alterações climáticas ocorridas no período proposto para o estudo.

A pesquisa foi aprovada pelo Comitê de Ética da Universidade Federal de São Carlos e seguiu as deliberações referentes à Resolução CNS 196/96.

\section{Análise dos Dados}

Com o número de óbitos e o número de residentes no município de São Carlos, foi possível calcular os coeficientes de mortalidade do município, por sexo, faixa etária (60 a 69 anos, 70 a 79 anos, 80 anos ou mais de idade) e causas de óbitos (número de óbitos no ano dividido pelo número de indivíduos da mesma faixa etária e de mesmo sexo residentes no município no ano, multiplicado por 1.000). Sobre os coeficientes de mortalidade obtidos, foi aplicado o teste de normalidade de Kolmogorov-Smirnov, que confirmou a distribuição normal de todo o conjunto de dados $(\mathrm{p}>0,05)$.

$\mathrm{O}$ teste $\mathrm{t}$ foi aplicado para verificar se havia diferenças entre os coeficientes de mortalidade dos homens e das mulheres e a ANOVA de fator único e o teste post hoc de Bonferroni foram utilizados para verificar diferenças entre os grupos etários.
O teste de Kolmogorov-Smirnov foi aplicado também aos dados de temperatura e umidade, e revelou que há distribuição normal $(\mathrm{p}>0,05)$. Dessa forma, pôde ser usado o teste de Pearson para verificar a presença de correlação entre essas variáveis meteorológicas e os coeficientes de mortalidade total e por causas de acordo com o CID-10.

O nível de significância adotado para todos os testes foi de 0,05 .

\section{Resultados}

\section{Características dos óbitos}

A Tabela 1 mostra o número total de óbitos no município de São Carlos ocorridos entre os anos de 1997 e 2006, em três faixas etárias (60 a 69 anos, 70 a 79 anos e 80 anos e mais de idade), e nos sexos masculino e feminino. A Tabela 2 mostra o número total de óbitos no período por raça e faixa etária.

A Tabela 3 mostra o número total de óbitos de indivíduos idosos ocorridos no município entre os anos de 1997 e 2006, por causas específicas do CID-10. É possível notar que as doenças do aparelho circulatório foram a causa mais frequente de óbitos em todos os anos estudados, mas o coeficiente de mortalidade por esta causa tem sofrido uma diminuição ao longo dos últimos anos. As neoplasias e os achados anormais em exames vêm se revezando na segunda posição entre as causas mais frequentes de mortalidade, seguidas pelas doenças do aparelho respiratório.

É possível observar na Tabela 4 as médias dos coeficientes de mortalidade total e por causas específicas do CID-10 dos anos de 1997 a 2006. Os homens apresentaram maiores coeficientes de mortalidade total, por doenças infecciosas e parasitárias, por neoplasias, por doenças dos aparelhos circulatório, respiratório, digestivo e geniturinário e por causas externas; enquanto as mulheres apresentaram maior coeficiente de mortalidade por doenças do sistema nervoso do que os homens.

Foi revelado que existe diferença significativa $(\mathrm{p}<0,002)$ entre três faixas etárias quanto à mortalidade total e por cada uma das causas de óbitos. A Tabela 5 evidenciou que para todas as causas de óbitos, havia diferenças significativas entre o grupo etário mais velho (80 anos e mais) e os outros dois mais novos. Para algumas causas de óbitos não houve diferença significativa entre o grupo etário de 60 a 69 anos e o grupo de 70 a 79 anos. 
A Tabela 6 mostra a média da umidade relati-

Tabela 1. Número de óbitos no município de São Carlos entre os anos de 1997 a 2006, por sexo e faixa etária.

\begin{tabular}{|c|c|c|c|}
\hline \multicolumn{4}{|c|}{ Óbitos em São Carlos } \\
\hline & Masculino & Feminino & Total \\
\hline \multicolumn{4}{|l|}{1997} \\
\hline Total & 429 & 392 & 821 \\
\hline 60 a 69 anos & 145 & 92 & 237 \\
\hline 70 a 79 anos & 168 & 122 & 290 \\
\hline 80 anos e mais & 116 & 178 & 294 \\
\hline \multicolumn{4}{|l|}{1998} \\
\hline Total & 399 & 356 & 755 \\
\hline 60 a 69 anos & 141 & 62 & 203 \\
\hline 70 a 79 anos & 143 & 125 & 268 \\
\hline 80 anos e mais & 115 & 169 & 284 \\
\hline \multicolumn{4}{|l|}{1999} \\
\hline Total & 412 & 417 & 829 \\
\hline 60 a 69 anos & 123 & 97 & 220 \\
\hline 70 a 79 anos & 153 & 137 & 290 \\
\hline 80 anos e mais & 136 & 183 & 319 \\
\hline \multicolumn{4}{|l|}{2000} \\
\hline Total & 419 & 408 & 827 \\
\hline 60 a 69 anos & 140 & 84 & 224 \\
\hline 70 a 79 anos & 142 & 127 & 269 \\
\hline 80 anos e mais & 137 & 197 & 334 \\
\hline \multicolumn{4}{|l|}{2001} \\
\hline Total & 422 & 379 & 801 \\
\hline 60 a 69 anos & 151 & 65 & 216 \\
\hline 70 a 79 anos & 151 & 132 & 283 \\
\hline 80 anos e mais & 120 & 182 & 302 \\
\hline \multicolumn{4}{|l|}{2002} \\
\hline Total & 452 & 389 & 841 \\
\hline 60 a 69 anos & 125 & 82 & 207 \\
\hline 70 a 79 anos & 164 & 115 & 279 \\
\hline 80 anos e mais & 163 & 192 & 355 \\
\hline \multicolumn{4}{|l|}{2003} \\
\hline Total & 396 & 446 & 842 \\
\hline 60 a 69 anos & 114 & 84 & 198 \\
\hline 70 a 79 anos & 155 & 146 & 301 \\
\hline 80 anos e mais & 127 & 216 & 343 \\
\hline \multicolumn{4}{|l|}{2004} \\
\hline Total & 432 & 410 & 842 \\
\hline 60 a 69 anos & 124 & 78 & 202 \\
\hline 70 a 79 anos & 177 & 125 & 302 \\
\hline 80 anos e mais & 131 & 207 & 338 \\
\hline \multicolumn{4}{|l|}{2005} \\
\hline Total & 424 & 399 & 823 \\
\hline 60 a 69 anos & 116 & 71 & 187 \\
\hline 70 a 79 anos & 169 & 137 & 306 \\
\hline 80 anos e mais & 139 & 191 & 330 \\
\hline \multicolumn{4}{|l|}{2006} \\
\hline Total & 485 & 438 & 923 \\
\hline 60 a 69 anos & 136 & 74 & 210 \\
\hline 70 a 79 anos & 200 & 139 & 339 \\
\hline 80 anos e mais & 149 & 225 & 374 \\
\hline
\end{tabular}

va do ar, e das temperaturas máxima, média e mínima de cada ano. Está exposto também o menor valor de umidade relativa mensal registrado em cada ano (Umidade relativa mínima), o maior valor de umidade relativa mensal registrado em cada ano (Umidade relativa máxima), o menor valor mensal de Temperatura mínima média de cada ano (Temperatura mínima mínima) e o maior valor mensal de temperatura máxima média de cada ano (Temperatura máxima máxima). As médias foram obtidas de acordo com os dados mensais disponíveis, sendo que não houve registro das médias da temperatura média e da média da temperatura máxima nos meses de março de 1999 e nos meses de setembro a novembro de 2000. Também não houve registro da média da temperatura mínima no mês de março de 1999. Quanto à umidade relativa média do ar, não houve dados nos meses de março de 1999, de maio até agosto de 2003, e de março de 2004.

Verificou-se a presença de correlação entre as variáveis meteorológicas e os coeficientes de mortalidade total e por causas de acordo com o CID10. A mortalidade total não apresentou correlação com as variáveis climáticas. As doenças infecciosas e parasitárias apresentaram correlação positiva significativa com a Umidade Mínima ( $\mathrm{r}$ $=0,642$ ) e correlação negativa com a Temperatura Mínima Mínima $(r=0,731)$. As doenças endócrinas, nutricionais e metabólicas apresentaram correlação negativa significativa com a Temperatura Mínima Mínima $(\mathrm{r}=0,690)$ e a Temperatura Máxima Máxima $(r=0,676)$. As doenças do aparelho respiratório apresentaram correlação negativa significativa com a Umidade Mínima $(r=0,669)$.

\section{Discussão}

Quanto à distribuição de óbitos por raça e estado civil, chama a atenção o grande número de registros das categorias "Ignorado", como é observado na Tabela 2. Essa categoria "Ignorado" foi elaborada pelo DATASUS e engloba indivíduos classificáveis em uma das outras categorias. Portanto, as outras categorias apresentam na verdade um número maior de indivíduos, que não nos é possível inferir. Assim, uma análise estatística inferencial dessas variáveis poderia não se aproximar da realidade e optamos por não realizá-la. No entanto, estudos conduzidos em outros países trazem informações sobre a influência dessas variáveis na mortalidade populaci- 
Tabela 2. Número de óbitos no município de São Carlos entre os anos de 1997 a 2006, por raça e estado civil.

\begin{tabular}{|c|c|c|c|c|c|c|c|c|c|c|c|}
\hline & 1997 & 1998 & 1999 & 2000 & 2001 & 2002 & 2003 & 2004 & 2005 & 2006 & Total \\
\hline \multicolumn{12}{|l|}{ Raça } \\
\hline Branca & 66 & 322 & 484 & 567 & 671 & 701 & 753 & 761 & 749 & 546 & 5620 \\
\hline Preta & 6 & 12 & 17 & 19 & 19 & 15 & 34 & 27 & 34 & 22 & 205 \\
\hline Parda & 3 & 9 & 15 & 20 & 26 & 24 & 12 & 19 & 31 & 28 & 187 \\
\hline Amarela & 35 & 2 & 26 & 65 & 1 & 10 & 12 & 10 & 5 & 3 & 169 \\
\hline Indígena & 0 & 0 & 2 & 6 & 0 & 0 & 0 & 0 & 0 & 0 & 8 \\
\hline Ignorada & 711 & 410 & 285 & 150 & 84 & 91 & 31 & 25 & 4 & 324 & 2115 \\
\hline \multicolumn{12}{|l|}{ Estado Civil } \\
\hline Solteiro & 88 & 74 & 70 & 68 & 86 & 112 & 227 & 246 & 152 & 70 & 1193 \\
\hline Casado & 365 & 344 & 334 & 379 & 342 & 304 & 195 & 235 & 312 & 315 & 3125 \\
\hline Viúvo & 337 & 317 & 308 & 327 & 301 & 283 & 199 & 187 & 277 & 289 & 2825 \\
\hline Separado judicialmente & 5 & 2 & 8 & 4 & 9 & 16 & 15 & 16 & 19 & 18 & 112 \\
\hline Outro & 8 & 15 & 9 & 15 & 3 & 2 & 0 & 1 & 0 & 3 & 56 \\
\hline Ignorado & 18 & 3 & 100 & 34 & 60 & 124 & 206 & 157 & 63 & 228 & 993 \\
\hline
\end{tabular}

Tabela 3. Número de óbitos em São Carlos, de acordo com o CID-10, por ano.

\begin{tabular}{|c|c|c|c|c|c|c|c|c|c|c|}
\hline \multirow[b]{2}{*}{ CID-10 } & \multicolumn{10}{|c|}{ Anos } \\
\hline & 1997 & 1998 & 1999 & 2000 & 2001 & 2002 & 2003 & 2004 & 2005 & 2006 \\
\hline Doenças do aparelho circulatório & 361 & 290 & 294 & 301 & 305 & 276 & 287 & 234 & 271 & 271 \\
\hline $\begin{array}{l}\text { Sintomas, sinais e achados anormais em } \\
\text { exames laboratoriais }\end{array}$ & 123 & 102 & 121 & 106 & 102 & 117 & 123 & 153 & 89 & 164 \\
\hline Neoplasias & 89 & 114 & 142 & 121 & 122 & 171 & 128 & 136 & 155 & 142 \\
\hline Doenças do aparelho respiratório & 80 & 83 & 108 & 100 & 109 & 129 & 132 & 142 & 102 & 128 \\
\hline $\begin{array}{l}\text { Doenças endócrinas, nutricionais e } \\
\text { metabólicas }\end{array}$ & 45 & 41 & 44 & 55 & 32 & 33 & 43 & 46 & 43 & 65 \\
\hline Doenças do aparelho digestivo & 40 & 33 & 44 & 40 & 39 & 44 & 41 & 39 & 50 & 44 \\
\hline Doenças do aparelho geniturinário & 27 & 28 & 23 & 19 & 29 & 26 & 21 & 17 & 20 & 16 \\
\hline Doenças infecciosas e parasitárias & 19 & 22 & 14 & 30 & 18 & 15 & 18 & 18 & 16 & 28 \\
\hline Causas externas & 16 & 8 & 19 & 22 & 14 & 15 & 20 & 20 & 20 & 19 \\
\hline Doenças do sistema nervoso & 13 & 13 & 8 & 19 & 10 & 6 & 11 & 15 & 31 & 35 \\
\hline Outras causas ${ }^{*}$ & 5 & 7 & 3 & 3 & 3 & 5 & 8 & 7 & 7 & 4 \\
\hline $\begin{array}{l}\text { Doenças do sangue e alguns transtornos } \\
\text { imunitários }\end{array}$ & 2 & 6 & 5 & 6 & 9 & 2 & 2 & 7 & 5 & 4 \\
\hline $\begin{array}{l}\text { Transtornos mentais e } \\
\text { comportamentais }\end{array}$ & 1 & 8 & 4 & 5 & 9 & 2 & 8 & 8 & 14 & 3 \\
\hline
\end{tabular}

*Doenças do olho e anexos, doenças do ouvido e da apófise mastóide, doenças da pele e do tecido subcutâneo, doenças do sistema osteomuscular e do tecido conjuntivo, malformação congênita e deformidades e anomalias cromossômicas, ou outras doenças fora do CID-10.

onal. Na onda de calor ocorrida no ano de 2003 na França, a mortalidade de indivíduos viúvos, solteiros e divorciados foi superior a mortalidade de indivíduos casados ${ }^{23}$ e, dos óbitos ocorridos numa onda de calor em Chicago no ano de 1995, estimou-se que houve 692 mortes e o risco de morte relacionada ao calor foi significativamente maior entre indivíduos negros ${ }^{24}$.
Em relação à causa da morte, é possível perceber que o envelhecimento populacional, que vem ocorrendo nas últimas décadas, está associado às alterações no perfil epidemiológico. Em 1930, cerca de $46 \%$ dos óbitos de idosos (60 anos ou mais) nas capitais brasileiras deviam-se a doenças infecto-parasitárias e 12\% eram devido a doenças do sistema circulatório ${ }^{25}$. Já em 1995 , 
Tabela 4. Coeficientes de mortalidade médios (1997 a 2006) nos grupos masculino e feminino e valor de $p$ para o teste t na comparação entre gêneros.

\begin{tabular}{|c|c|c|c|}
\hline Mortalidade & Média & Desvio-padrão & Valor de $p$ \\
\hline Total & & & $<0,001$ \\
\hline Homens & 47,37 & 3,52 & \\
\hline Mulheres & 35,09 & 2,92 & \\
\hline Doenças infecciosas e parasitárias & & & 0,038 \\
\hline Homens & 1,13 & 0,40 & \\
\hline Mulheres & 0,84 & 0,25 & \\
\hline Neoplasias & & & $<0,001$ \\
\hline Homens & 8,42 & 1,22 & \\
\hline Mulheres & 4,80 & 0,83 & \\
\hline Doenças do sangue e alguns transtornos imunitários & & & 0,987 \\
\hline Homens & 0,23 & 0,16 & \\
\hline Mulheres & 0,23 & 0,10 & \\
\hline Doenças endócrinas, nutricionais e metabólicas & & & 0,111 \\
\hline Homens & 1,90 & 0,50 & \\
\hline Mulheres & 2,39 & 0,70 & \\
\hline Transtornos mentais e comportamentais & & & 0,532 \\
\hline Homens & 0,32 & 0,25 & \\
\hline Mulheres & 0,28 & 0,17 & \\
\hline Doenças do sistema nervoso & & & 0,001 \\
\hline Homens & 0,59 & 0,38 & \\
\hline Mulheres & 0,83 & 0,48 & \\
\hline Doenças do aparelho circulatório & & & $<0,001$ \\
\hline Homens & 15,87 & 3,00 & \\
\hline Mulheres & 12,92 & 2,68 & \\
\hline Doenças do aparelho respiratório & & & $<0,001$ \\
\hline Homens & 6,81 & 1,04 & \\
\hline Mulheres & 4,26 & 1,06 & \\
\hline Doenças do aparelho digestivo & & & $<0,001$ \\
\hline Homens & 2,52 & 0,36 & \\
\hline Mulheres & 1,62 & 0,28 & \\
\hline Doenças do aparelho genitourinário & & & 0,029 \\
\hline Homens & 1,31 & 0,44 & \\
\hline Mulheres & 0,97 & 0,30 & \\
\hline Sintomas, sinais e achados anormais & & & 0,004 \\
\hline Homens & 6,75 & 1,32 & \\
\hline Mulheres & 5,12 & 1,15 & \\
\hline Causas externas & & & $<0,001$ \\
\hline Homens & 1,21 & 0,30 & \\
\hline Mulheres & 0,54 & 0,22 & \\
\hline Outras causas & & & 0,218 \\
\hline Homens & 0,21 & 0,07 & \\
\hline Mulheres & 0,28 & 0,15 & \\
\hline
\end{tabular}

essa porcentagem foi invertida: 7\% dos óbitos ocorriam por doenças infecto-parasitárias e 33\% por doenças do sistema circulatório. Paes ${ }^{26}$ analisou a mortalidade de indivíduos acima dos 65 anos de idade no Brasil no período de 1980 a 1995 e verificou que as doenças do aparelho circulatório e as neoplasias foram as causas de mortes mais frequentes. Já Vasconcelos ${ }^{27}$ analisou a mortalidade da população idosa no perío- do de 1990 a 2001 e verificou aumento da longevidade com predomínio de morte por doenças do aparelho circulatório, seguido pelas doenças do aparelho respiratório e neoplasmas. Em nosso estudo, as doenças do aparelho circulatório também foram a principal causa de óbito, porém, no segundo lugar, alternaram-se as causas "sinais, sintomas e achados anormais em exames laboratoriais" e "neoplasias". As doenças do 
Tabela 5. Média e desvios-padrão dos coeficientes de mortalidade anuais (1997 a 2006), por faixas etárias e valores de $p$ para ANOVA de fator único (com post hoc de Bonferroni).

\begin{tabular}{|c|c|c|c|c|c|c|}
\hline & \multirow[b]{2}{*}{$\begin{array}{l}\text { Faixa } \\
\text { etária }\end{array}$} & \multirow[b]{2}{*}{ Média } & \multirow[b]{2}{*}{$\begin{array}{l}\text { Desvio- } \\
\text { padrão }\end{array}$} & \multicolumn{2}{|c|}{$\begin{array}{c}\text { Intervalo de } \\
\text { confiança para a } \\
\text { média a } 95 \%\end{array}$} & \multirow[b]{2}{*}{$\begin{array}{l}\text { Valor } \\
\text { de } p\end{array}$} \\
\hline & & & & $\begin{array}{l}\text { Limite } \\
\text { inferior }\end{array}$ & $\begin{array}{l}\text { Limite } \\
\text { superior }\end{array}$ & \\
\hline \multirow[t]{3}{*}{ Todas as doenças } & 60 a 69 & 18,53 & 2,29 & 16,89 & 20,17 & \multirow[t]{3}{*}{$<\mathbf{0 , 0 0 1}$} \\
\hline & 70 a 79 & 45,07 & 4,53 & 41,83 & 48,31 & \\
\hline & 80 e mais & 125,38 & 9,44 & 118,62 & 132,13 & \\
\hline \multirow[t]{3}{*}{ Doenças infecciosas e parasitárias } & 60 a $69^{*}$ & 0,49 & 0,17 & 0,36 & 0,61 & \multirow[t]{3}{*}{$<0,001$} \\
\hline & 70 a $79^{*}$ & 1,20 & 0,51 & 0,83 & 1,57 & \\
\hline & 80 e mais & 2,49 & 1,30 & 1,56 & 3,42 & \\
\hline \multirow[t]{3}{*}{ Neoplasias } & 60 a 69 & 4,05 & 0,62 & 3,60 & 4,49 & \multirow[t]{3}{*}{$<0,001$} \\
\hline & 70 a 79 & 7,94 & 1,39 & 6,94 & 8,93 & \\
\hline & 80 e mais & 12,80 & 3,83 & 10,06 & 15,53 & \\
\hline \multirow{3}{*}{$\begin{array}{l}\text { Doenças do sangue e alguns transtornos } \\
\text { imunitários }\end{array}$} & 60 a $69^{*}$ & 0,10 & 0,10 & 0,03 & 0,17 & \multirow[t]{3}{*}{$<0,001$} \\
\hline & 70 a $79^{*}$ & 0,22 & 0,19 & 0,08 & 0,35 & \\
\hline & 80 e mais & 0,85 & 0,47 & 0,51 & 1,18 & \\
\hline \multirow{3}{*}{$\begin{array}{l}\text { Doenças endócrinas, nutricionais e } \\
\text { metabólicas }\end{array}$} & 60 a $69^{*}$ & 1,06 & 0,39 & 0,78 & 1,34 & \multirow[t]{3}{*}{$<0,001$} \\
\hline & 70 a $79^{*}$ & 2,23 & 0,59 & 1,81 & 2,65 & \\
\hline & 80 e mais & 6,93 & 2,88 & 4,88 & 8,99 & \\
\hline \multirow[t]{3}{*}{ Transtornos mentais e comportamentais } & 60 a $69^{\star}$ & 0,09 & 0,12 & 0,01 & 0,18 & \multirow[t]{3}{*}{$<0,001$} \\
\hline & 70 a $79^{*}$ & 0,19 & 0,17 & 0,06 & 0,31 & \\
\hline & 80 e mais & 1,45 & 1,01 & 0,73 & 2,18 & \\
\hline \multirow[t]{3}{*}{ Doenças do sistema nervoso } & 60 a $69^{\star}$ & 0,23 & 0,12 & 0,15 & 0,31 & \multirow[t]{3}{*}{$<0,001$} \\
\hline & 70 a $79^{*}$ & 0,85 & 0,58 & 0,43 & 1,26 & \\
\hline & 80 e mais & 2,88 & 1,84 & 1,56 & 4,19 & \\
\hline \multirow[t]{3}{*}{ Doenças do aparelho circulatório } & 60 a 69 & 5,78 & 1,56 & 4,66 & 6,90 & \multirow[t]{3}{*}{$<0,001$} \\
\hline & 70 a 79 & 16,71 & 3,90 & 13,92 & 19,49 & \\
\hline & 80 e mais & 45,21 & 8,42 & 39,19 & 51,24 & \\
\hline \multirow[t]{3}{*}{ Doenças do aparelho respiratório } & 60 a 69 & 1,68 & 0,31 & 1,45 & 1,90 & \multirow[t]{3}{*}{$<0,001$} \\
\hline & 70 a 79 & 5,81 & 0,63 & 5,36 & 6,26 & \\
\hline & 80 e mais & 20,44 & 4,25 & 17,40 & 23,47 & \\
\hline \multirow[t]{3}{*}{ Doenças do aparelho digestivo } & 60 a 69 & 1,33 & 0,35 & 1,08 & 1,58 & $<0,001$ \\
\hline & 70 a 79 & 2,15 & 0,52 & 1,78 & 2,53 & \\
\hline & 80 e mais & 4,67 & 0,74 & 4,14 & 5,20 & \\
\hline Doenças do aparelho geniturinário & 60 a $69^{*}$ & 0,45 & 0,26 & 0,26 & 0,63 & $<0,001$ \\
\hline & 70 a $79^{\star}$ & 1,19 & 0,52 & 0,82 & 1,57 & \\
\hline & 80 e mais & 3,88 & 1,35 & 2,91 & 4,84 & \\
\hline Sintomas, sinais e achados anormais & 60 a 69 & 2,60 & 0,60 & 2,17 & 3,04 & $<0,001$ \\
\hline & 70 a 79 & 5,51 & 1,53 & 4,41 & 6,60 & \\
\hline & 80 e mais & 20,86 & 3,78 & 18,16 & 23,57 & \\
\hline Causas externas & 60 a $69^{\star}$ & 0,54 & 0,26 & 0,35 & 0,73 & $<0,001$ \\
\hline & 70 a $79^{*}$ & 0,83 & 0,41 & 0,54 & 1,13 & \\
\hline & 80 e mais & 2,13 & 0,80 & 1,55 & 2,70 & \\
\hline Outras causas & 60 a $69^{*}$ & 0,13 & 0,10 & 0,06 & 0,20 & 0,001 \\
\hline & 70 a $79^{*}$ & 0,26 & 0,23 & 0,09 & 0,42 & \\
\hline & 80 e mais & 0,79 & 0,55 & 0,40 & 1,18 & \\
\hline
\end{tabular}

${ }^{*}$ ) Grupos com símbolos iguais não apresentaram diferença significativa entre si, de acordo com o teste post hoc de Bonferroni.

aparelho respiratório ocuparam o quarto lugar entre as principais causas de óbitos.

Segundo a Organização Mundial da Saúde ${ }^{28}$, as doenças cardiovasculares são a principal cau- 
Tabela 6. Médias das variáveis climáticas no município de São Carlos (SP) no período de 1997 a 2006.

\begin{tabular}{ccccccccc}
\hline Ano & $\begin{array}{c}\text { Umidade } \\
\text { relativa } \\
\text { média }\end{array}$ & $\begin{array}{c}\text { Umidade } \\
\text { mínima }\end{array}$ & $\begin{array}{c}\text { Umidade } \\
\text { máxima }\end{array}$ & $\begin{array}{c}\text { Temperatura } \\
\text { média da } \\
\text { mínima }\end{array}$ & $\begin{array}{c}\text { Temperatura } \\
\text { média da } \\
\text { máxima }\end{array}$ & $\begin{array}{c}\text { Temperatura } \\
\text { média } \\
\text { compensada }\end{array}$ & $\begin{array}{c}\text { Temperatura } \\
\text { mínima } \\
\text { mínima }\end{array}$ & $\begin{array}{c}\text { Temperatura } \\
\text { máxima } \\
\text { máxima }\end{array}$ \\
\hline 1997 & 73,7 & 58 & 86 & 15,8 & 27,3 & 20,4 & 11,5 \\
1998 & 75,3 & 67 & 85 & 16,1 & 27,4 & 20,6 & 11,3 \\
1999 & 71,5 & 56 & 82 & 15,3 & 27,3 & 20,2 & 11,6 \\
2000 & 73,2 & 65 & 84 & 15,8 & 27,0 & 20,0 & 10,1 \\
2001 & 72,3 & 59 & 79 & 16,1 & 27,6 & 20,7 & 12,6 \\
2002 & 69,4 & 55 & 81 & 16,7 & 28,3 & 21,4 & 12,1 \\
2003 & 71,6 & 56 & 84 & 15,9 & 27,6 & 20,8 & 30,1 \\
2004 & 73,1 & 48 & 84 & 15,3 & 26,7 & 20,0 & 30,3 \\
2005 & 72,8 & 55 & 87 & 16,6 & 27,2 & 20,7 & 11,3 \\
2006 & 70,5 & 54 & 81 & 16,1 & 27,5 & 20,8 & 12,1 \\
\hline
\end{tabular}

mento, estima-se que, em 2020, um terço de todos os óbitos será devido às doenças cardiovasculares. A redução das taxas de mortalidade por doenças do aparelho circulatório tem sido observada em vários países do mundo desenvolvido, como consequência de mudanças no estilo de vida e melhoria da tecnologia para prevenção e tratamento dessas doenças. Com base nos dados deste estudo, é possível perceber que em São Carlos essa tendência também tem sido observada: passou de 361 óbitos em 1997 para 271 em 2006, uma redução de $25 \%$.

Gadelha e Martins ${ }^{29}$ relatam que os óbitos devido à neoplasia cresceram nos últimos anos, já que o envelhecimento relaciona-se diretamente com o desenvolvimento das neoplasias e que, com o passar do tempo, diferentes agressões externas geram danos ao DNA das células, possibilitando o desenvolvimento das primeiras células geradoras dos tumores malignos. Boing et al. ${ }^{30}$ descreveram a morbidade hospitalar e a mortalidade por neoplasias no Brasil e observaram que, entre 2002 e 2004, ocorreram 405.415 óbitos por neoplasias no país. As maiores taxas de mortalidade foram identificadas nas regiões Sul $(20,96 \%)$ e Sudeste (52,13\%), concluindo-se que a taxa de neoplasias no Brasil é elevada, o que está em concordância com os achados do presente estudo em São Carlos.

Em relação ao gênero, o presente estudo observou maior número de óbitos em indivíduos do sexo masculino em todas as causas específicas do CID-10, exceto na mortalidade por doenças do sistema nervoso, na qual as mulheres apresentaram maior coeficiente de mortalidade. Laurenti et al. ${ }^{31}$ também relatam que, exceto para as doenças próprias ou específicas do gênero, os coeficientes masculinos são maiores em praticamente todas as causas, sendo tal fato observado em todas as idades. A diminuição da mortalidade por causas maternas, a procura maior por serviços de saúde por parte das mulheres facilitando o diagnóstico e o tratamento precoce das doenças e as diferenças biológicas consideradas como protetoras contribuem para que as mulheres vivam mais do que os homens. Os riscos ambientais e ocupacionais, como acidentes de trabalho, de trânsito, homicídios e o estresse associado às mudanças socioeconômicas contribuem para a maior mortalidade entre os homens idosos $^{32}$. Benyamini et al. ${ }^{33}$ e Deeg e Bath ${ }^{34}$ relatam que o hábito de fumar e o consumo de álcool foi maior entre os homens que entre as mulheres da população idosa estudada, mas essa relação pode se igualar ou se inverter futuramente devido às mudanças nos hábitos e comportamentos femininos.

Lima-Costa et al. ${ }^{35}$ constataram que as taxas de mortalidade por doenças do aparelho circulatório, neoplasias, doenças do aparelho respiratório, causas externas, doenças infecciosas e parasitárias e sintomas, sinais e afecções maldefinidos aumentaram gradualmente com a idade. Como era esperado, o presente estudo constatou que, para todas as causas de óbitos, houve diferenças significativas entre o grupo etário mais velho (80 anos e mais) e os outros dois mais novos, sendo que quanto maior a faixa etária, maior a mortalidade.

O presente estudo verificou algumas relações entre as variáveis climáticas e a mortalidade no município de São Carlos que refletiram, em diferente grau, algumas tendências observadas em outros estudos de origens geográficas diversas. 
Bell et al. ${ }^{36}$ investigaram a mortalidade relacionada ao calor em três cidades latino-americanas: Cidade do México (México), São Paulo (Brasil) e Santiago, Chile) using a case-crossover approach for 754 291Santiago (Chile). We considered lagged exposures, confoundingOs autores concluíram que as temperaturas elevadas estão associadas com o risco de mortalidade risk in these Latin American cities, with the strongest associationsnessas cidades, sendo a associação mais forte em São Paulo (a mais quente das três cidades estudadas).

Parece que os idosos são mais sensíveis aos efeitos da alta temperatura do que indivíduos em outras faixas etárias. Em estudo realizado entre os anos de 1986 a 1997, por Linares e Diaz ${ }^{37}$, no qual se analisou os óbitos ocorridos em Madri (Espanha), foi revelado que existe associação entre calor e mortalidade. No grupo etário de 18 a 64 anos o aumento da mortalidade permanece praticamente constante a cada aumento de grau da temperatura máxima diária, enquanto há aumento significativo da mortalidade no grupo das pessoas com mais de 65 anos. No Condado de Maricopa (Arizona, EUA), de junho a setembro de 2000 a 2005, foram notificadas 136 mortes (idade média de 56 anos) relacionadas com o calor. Semelhantemente, as taxas de mortalidade ajustada para idade foram maiores entre os indivíduos com 75 anos ou mais de idade ${ }^{38}$.

Paixão e Nogueira ${ }^{39}$ publicaram os resultados da pesquisa realizada por eles a fim de estimar as consequências das ondas de calor em Portugal entre 1990 e 1992. Os autores observaram que, dentre as causas de morte associadas a esse fenômeno, destacaram-se as doenças do aparelho circulatório, as neoplasias malignas, as doenças do aparelho respiratório e o grupo dos sintomas, sinais e afecções mal definidas. Já em nosso estudo, a mortalidade por doenças infecciosas e parasitárias e por doenças endócrinas, nutricionais e metabólicas apresentou correlações negativas com variáveis de temperatura, indicando que ocorrem mais mortes por essas doenças em anos com temperaturas mais baixas.

O impacto da temperatura ambiente sobre a mortalidade no período de 1991 a 1994, em São Paulo, foi analisado por Gouveia et al..$^{40}$, que observaram a influência tanto de altas quanto de baixas temperaturas sobre a mortalidade. Os autores constataram que, entre os idosos, ocorreu um aumento de 2,6\% em todas as causas de mortalidade por grau de aumento na temperatura acima de $20^{\circ} \mathrm{C}$ e $5,5 \%$ de aumento da mortalidade por grau quando a temperatura cai abaixo de $20^{\circ} \mathrm{C}$.
Em países desenvolvidos, a mortalidade é maior no inverno. Muitas causas têm sido sugeridas, como o ciclo luz/escuridão, temperatura/ clima, e agentes infecciosos. Reichert et al. ${ }^{41}$ analisaram a mortalidade mensal nos EUA entre 1959 e 1999 e concluíram que o maior determinante do aumento da mortalidade no inverno é o vírus influenza. Nakaji et al. ${ }^{42}$ também investigaram a influência da variação sazonal sobre a mortalidade causada por algumas doenças no Japão e perceberam que no inverno, há maiores índice de óbitos para doenças infecciosas e parasitárias (incluindo tuberculose, doenças respiratórias, pneumonia e influenza), diabetes e doenças digestivas. Já as neoplasias não foram influenciadas pela sazonalidade.

$\mathrm{Na}$ Inglaterra e no País de Gales há aproximadamente 20 mil mortes em excesso por doenças cardiovasculares a cada inverno. Woodhouse et al. $^{43}$ sugerem que a variação sazonal no fibrinogênio pode ser induzido pelas infecções respiratórias via ativação da fase aguda da resposta inflamatória. As variações sazonais nos fatores de risco cardiovasculares do fibrinogênio e fator de coagulação VII causados pela influenza podem prover uma possível explicação para a marcante variação sazonal nas mortes por isquemia cardíaca e por acidente vascular encefálico entre idosos.

No presente estudo não houve correlação entre temperatura e mortalidade por doenças respiratórias, havendo apenas correlação negativa entre umidade mínima e mortalidade por doenças respiratórias. Entretanto, alguns estudos observaram correlação entre mortes por causas respiratórias e altas temperaturas ${ }^{44-46}$. Oztuna et al. ${ }^{47}$ estudaram a variação sazonal, em termos de pressão atmosférica, umidade e temperatura na incidência de tromboembolismo pulmonar em 206 pacientes com diagnóstico de embolia pulmonar, entre junho de 2001 e maio de 2006. Foi encontrada uma correlação positiva entre pressão atmosférica e umidade e incidência de casos.

A maior parte dos estudos feitos sobre a relação entre clima e mortalidade se refere àquela causada por problemas cardiovasculares. Vários autores encontraram associação entre baixas temperaturas e mortes por acidentes vasculares ${ }^{48-}$ 52; enquanto alguns outros encontraram associação com altas temperaturas ${ }^{53,54}$. Porém em São Carlos, não foi observada relação entre mortalidade por doenças cardiovasculares e fatores climáticos.

Dentre as limitações do estudo estão os seguintes fatores: especificidade geográfica do local 
estudado (São Carlos) e ausência de identificação de dados meteorológicos máximos e mínimos, uma vez que só foram fornecidos dados máximos médios e mínimos médios.

Foi possível notar que há predominância de mortes de indivíduos na faixa dos 80 anos ou mais, do sexo masculino e que doenças do aparelho circulatório foram as principais causas de óbito. Foi possível concluir que houve relação entre o clima e a mortalidade de idosos em São Carlos no período de 1997 a 2006. Esta associação foi observada na correlação positiva entre mortalidade por doenças infecciosas e Umidade Mínima, e correlação negativa entre mortalidade por doenças infecciosas e Temperatura Mínima Mínima, entre mortalidade por doenças respiratórias e Umidade Mínima, e entre mortalidade por doenças endócrinas e Temperatura Mínima Mínima e Temperatura Máxima Máxima.

\section{Colaboradores}

FV Soares, JR Rebelatto e P Greve trabalharam na concepção do projeto e na redação do texto. FA Sendín, BG Benze e AP Castro trabalharam na análise dos dados e na redação final.

\section{Agradecimento}

Os autores agradecem à Fundação de Amparo à Pesquisa do Estado de São Paulo (FAPESP) pelo apoio financeiro. 


\section{Referências}

1. Pearce F. O Aquecimento Global Causas e Efeitos de um Mundo mais Quente. Série Mais Ciência. São Paulo: Publifolha; 2002.

2. IPCC Working Group II Contribution to the Intergovernmental Panel on Climate Change Fourth Assessment Report. Climate Change 2001: The Scientific Basis. Cambridge: Cambridge University Press; 2001.

3. IPCC Working Group II Contribution to the Intergovernmental Panel on Climate Change Fourth Assessment Report. Climate Change 2007: Climate Change Impacts, Adaptation and Vulnerability. Summary for Policymakers. Cambridge: Cambridge University Press; 2007.

4. Mendonça F. Aspectos da interação clima-ambientesaúde humana: da relação sociedade-natureza à (in)sustentabilidade ambiental. Curitiba: Ed. Universidade Federal do Paraná; 2000.

5. Haines A. Implicações para a saúde. In: Legget J, organizador. Aquecimento global - o relatório do Greenpeace. Rio de Janeiro: Fund. Getúlio Vargas; 1992.

6. Roberts LK, Samlowski WE, Daynes RA. The immunological consequences of ultraviolet radiation exposure. Photo Dermat 1986; 3(5):284-298.

7. Beltrando G, Chémery L. Dictionnaire du climat. Paris: Larousse; 1995.

8. Guyton AC, Hall JE. Tratado de fisiologia médica. 10a ed. Rio de Janeiro: Guanabara Koogan; 2002.

9. Kenney WL, Munce TA. Invited Review: Aging and human temperature regulation. J Appl Physiol 2003; 95(6):2598-2603

10. Inoue Y, Kuwabara T, Araki T. Maturation and agingrelated changes in heat loss effector function. J Physiol Antropol Appl Human Sci 2004; 23(6):289-294.

11. Spirduso WW. Dimensões físicas do envelhecimento. São Paulo: Manole; 2005.

12. Havenith G. Temperature regulation and technology. Gerontechnology 2001; 1(1):41-49.

13. Basu R, Samet JM. An exposure assessment study of ambient temperature and mortality: a review of the epidemiologic evidence. Epidemiol Rev 2002; 24(2):190-202.

14. Ellis FP, Nelson F. Mortality in the elderly in a heat wave in New York City, august 1975. Environ Res 1978; 15(3):504-512.

15. Macfarlane A. Daily mortality and environment in English conurbations. II. Deaths during summer hot spells in Greater London. Environ Res 1978; 15(3):332-334.

16. Ellis FP, Prince HP, Lovatt G, Whittington RM. Mortality and Morbidity in Birmingham during the 1976 heat wave. Q J Med 1980; 49(193):1-8.

17. Calado R, Nogueira PJ, Catarino J, Paixão EJ, Botelho J, Carreira M, Falcão JM. A onda de calor de Agosto de 2003 e os seus efeitos sobre a mortalidade da população portuguesa. Rev Portug Saúde Pub 2004; 22(2):7-20.

18. Conti S, Meli P, Minelli G, Solimini R, Toccaceli V, Vichi M, Beltrano C, Perini L. Epidemiologic study of mortality during the summer 2003 heat wave in Italy. Environ Res 2005; 98(3):390-399.
19. Kyselý J, Kríz B. Decreased impacts of the 2003 heat waves on mortality in the Czech Republic: an improved response? Int J Biometeorol 2008; 52(8):733745.

20. Knowlton K, Rotkin-Ellman M, King G, Margolis HG, Smith D, Solomon G, Trent R, English P. The 2006 California Heat Wave: Impacts on Hospitalizations and Emergency Department Visits. Environm Health Perspec 2009; 117(1):61-67.

21. Stöllberger C, Lutz W, Finsterer J. Heat-related sideeffects of neurological and non-neurological medication may increase heatwave fatalities. Europ J Neurol 2009; 16(7):879-882.

22. Brooke AG, Bell ML. Weather-Related Mortality: How Heat, Cold, and Heat Waves Affect Mortality in the United States. Epidem 2009; 20(2):205-213.

23. Fouillet A, Rey G, Laurent F, Pavillon G, Bellec S, Guihenneuc-Jouyaux C, Clavel J, Jougla E, Hémon D. Excess mortality related to the August 2003 heat wave in France. Intern Arch Environ Occup Health 2006; 80(1):16-24.

24. Semenza JC, Rubin CH, Falter KH, Selanikio JD, Flanders DW, Howe HL, Wilhelm JL. Heat related deaths during the July 1995 heat wave in Chicago. $N$ Engl J Med 1996; 335(2):84-90.

25. Prata PR. A Transição Epidemiológica no Brasil. Cad Saude Publica 1992; 8(2):168-175.

26. Paes NA. A Geografia da mortalidade por causas dos idosos no Brasil; 2000. [acessado 2008 out 27]. Disponível em: www.abep.nepo.unicamp.br/docs/ anais/pdf/2000/Todos/saut18_3.pdf

27. Vasconcelos AMN. Causas de morte em idosos no Brasil. XIV Encontro Nacional de Estudos Populacionais, ABEP, 2004 [acessado 2008 out 27]. Disponível em: www.abep.nepo.unicamp.br/site_eventos_ abep/PDF/ABEP2004_664.pdf

28. Organização Mundial de Saúde (OMS). Ageing and Life Course. Department of Noncommunicable Diseases Prevention and Health Promotion. Noncommunicable Diseases and Mental Health Cluster. Life course perspectives on coronary heart disease, stroke and diabetes. Geneva: Organização Mundial de Saúde (OMS); 2001.

29. Gadelha MIP, Martins RG. Neoplasias no idoso. In: Freitas EV, Py L, Néri AL, Cançado FAX, Gorzoni ML, Rocha SM, organizadores. Tratado de geriatria e gerontologia. Rio de Janeiro: Guanabara Koogan; 2002.

30. Boing AF, Vargas SAL, Boing AC. A carga das neoplasias no Brasil: mortalidade e morbidade hospitalar entre 2002-2004. Rev Assoc Med Bras 2007; 53(4):317-322.

31. Laurenti R, Jorge MHPM, Gotlieb SLD. Perfil epidemiológico da morbi-mortalidade masculina. Cien Saude Colet 2005; 10(1):35-46.

32. Rede Interagencial de Informações para a Saúde (RIPSA). Indicadores e Dados Básicos - 2003. Brasília: Rede Interagencial de Informações para a Saúde (RIPSA) (folder); 2004 
33. Benyamini Y, Blumstein T, Lusky A, Modan B. Gender differences in the self-rated health-mortality association: is it poor self-rated health that predicts mortality or excellent self-rated health that predicts survival? Gerontologist 2003; 43(3):396-405.

34. Deeg DJH, Bath PA. Self-rated health, gender, and mortality in older persons: introduction to a special section. Gerontologist 2003; 43(3):369-371.

35. Lima-Costa MF, Peixoto SV, Giatti L. Tendências da mortalidade entre idosos brasileiros (1980-2000). Epidemiol Serv Saúde 2004; 13(4):217-228.

36. Bell M, O’Neill MS, Ranjit N, Borja-Aburto VH, Cifuentes LA, Gouveia NC. Vulnerability to heatrelated mortality in Latin America: a case-crossover study in São Paulo, Brazil, Santiago, Chile and Mexico City, Mexico. Intern J Epidem 2008; 37(4): 796-804.

37. Linares C, Diaz J. Impact of high temperatures on hospital admissions: comparative analysis with previous studies about mortality (Madrid). Europ J Pub Health 2008; 18(3):317-322.

38. Yip FY, Flanders WD, Wolkin A, Engelthaler D, Humble W, Neri A, Lewis L, Backer L, Rubin C. The impact of excess heat events in Maricopa County, Arizona: 2000-2005. Int J Biometeorol 2008; 52(8) 765-772.

39. Paixão EJ, Nogueira PJ. Efeitos de uma onda de calor na mortalidade. Rev Portug Saúde Pública 2003; 21(1):41-54

40. Gouveia N, Hajat S, Armstrong B. Socioeconomic differentials in the temperature-mortality relationship in São Paulo, Brazil. Internat J Epidemiol 2003; 32(3):390-397.

41. Reichert TA, Simonsen L, Sharma A, Pardo SA, Fedson DS, Miller MA. Influenza and the winter increase in mortality in the United States, 1959. 1999. Am J Epidemiol 2004; 160(5):492-502.

42. Nakaji S, Parodi S, Fontana V, Umeda T, Suzuki K, Sakamoto J, Fukuda S, Wada S, Sugawara K. Seasonal changes in mortality rates from main causes of death in Japan (1970-1999). Eur J Epidemiol 2004 10(19):905-913

43. Woodhouse PR, Khaw KT, Plummer M, Foley A, Meade TW. Seasonal variations of plasma fibrinogen and factor VII activity in the elderly: winter infections and death from cardiovascular disease. Lancet 1994; 343(8895):435-439.

44. Hajat S, Armstrong BG, Gouveia N, Wilkinson P. Mortality displacement of heat-related deaths: comparison of Delhi, São Paulo, and London. Epidemiol 2005; 16(5):613-620.

45. Baccini M, Biggeri A, Accetta G, Kosatsky T, Katsouyanni K, Analitis A, Anderson HR, Bisanti L, D'Ippoliti D, Danova J, Forsberg B, Medina S, Paldy A, Rabczenko D, Schindler C, Michelozzi P. Heat Effects on Mortality in 15 European Cities. Epidemiol 2008; 19(5):711-719.
46. Michelozzi P, Accetta G, De Sario M, D’Ippoliti D, Marino C, Baccini M, Biggeri A, Anderson HR, Katsouyanni K, Ballester F, Bisanti L, Cadum E, Forsberg B, Forastiere F, Goodman PG, Hojs A, Kirchmayer U, Medina S, Paldy A, Schindler C, Sunyer J, Perucci CA; PHEWE Collaborative Group. High Temperature and Hospitalizations for Cardiovascular and Respiratory Causes in 12 European Cities. Amer J Respir Crit Care Med 2009; 179(5):383-389.

47. Oztuna F, Ozsu S, Topba ${ }^{\circ}$ M, Bulbul Y, $\mathrm{Ko}^{\circ} \mathrm{ucu}$ P, Ozlu T. Meteorological parameters and seasonal variations in pulmonary thromboembolism. Amer J Emerg Med 2008; 26(9):1035-1041.

48. Medina-Ramon M, Zanobetti A, Cavanagh DP, Schwartz J. Extreme temperatures and mortality: Assessing effect modification by personal characteristics and specific cause of death in a Multi-City case-only analysis. Environm Health Perspec 2006; 114(9):1331-1336.

49. Gonçalves FLT, BraunS, Dias PLS, Sharovsky R. Influences of the weather and air pollutants on cardiovascular disease in the metropolitan area of São Paulo. Environ Res 2007; 104(2):275-281.

50. Rufca GF, Zaffani E, Zerbini R, Gaia FFP, Oliveira FN, Tognolla WA. Influência das variações circadianas e de temperatura no AVEi. Rev Assoc Med Bras 2009; 55(1):60-63.

51. Kysely J, Pokorna L, Kyncl J, Kriz B. Excess cardiovascular mortality associated with cold spells in the Czech Republic. BMC Public Health 2009; 9:19.

52. Ha J, Yoona J, Kimb H. Relationship between winter temperature and mortality in Seoul, South Korea, from 1994 to 2006. Sci Total Environ 2009; 407(7):158-216

53. Barnett AG. Temperature and cardiovascular deaths in the US elderly changes over time. Epidem 2007; 18(3):369-372.

54. Bi P, Parton KA, Wang J, Donald K. Temperature and direct effects on population health in Brisbane, 1986-1995. J Environ Health 2008; 70(8):48-53.

Artigo apresentado em 25/10/2009

Aprovado em 21/02/2010

Versão final apresentada em 15/03/2010 